\begin{tabular}{|c|c|c|c|}
\hline Eiszeitalter u. Gegenwart & 44 & $\begin{array}{l}94-105 \\
10 \text { Abb.. }\end{array}$ & Hannover 1994 \\
\hline
\end{tabular}

\title{
Gravitative Deformationserscheinungen aus dem Älteren Quartär der Leipziger Tieflandsbucht
}

\author{
Frank Wolfgang Junge, Reinhardt Baudenbacher, Lothar Eissmann*)
}

Quaternary, Tertiary, Elsterian glaciation, Cryogenic gravitative deformation, Germany.

\begin{abstract}
Kurzfassung: Am Beispiel eines im mitteldeutschen Braunkohlentagebau Schleenhain im Übergangsbereich Quartär/Tertiär aufgeschlossenen Profils werden typische genetische Formen kryogen verursachter gravitativer Deformationen aus dem Anaglazial der Elstervereisung des mitteldeutschen Raumes beschrieben. Sie zeigen einen mehrfachen Wechsel von Dauergefrornis und Frostbodendegradation der periglaziären Sedimentunterlage im Vorfeld des herannahenden Inlandeises für diesen Zeitraum an.
\end{abstract}

\section{[Impressive gravitative deformation structures from the Older Quaternary of the "Leipziger Tieflandsbucht"]}

\begin{abstract}
An example of a geological profile with Quaternary-Tertiary sequences from the open cast lignite mine Schleenhain with typical genetic forms of cryogenic gravitative deformation structures of the anaglacial period of the Elsterian glaciation in Central Germany is described. It shows a repeated change of frost and permafrost degradation periods in periglacial sediments in front of the ice sheet
\end{abstract}

\section{Einleitung und Überblick}

Der im Bereich der Nordwestsächsischen Tiefscholle gelegene Braunkohlentagebau Schleenhain südlich Leipzig zählt zu den geologisch bedeutendsten künstlichen Aufschlüssen im Känozoikum Mitteleuropas (Abb. 1).

\footnotetext{
*) Anschriften der Verfasser: Dr. F.W. Junge, Universität Leipzig, Quartärzentrum, Arbeitsgruppe "Paläoklimatologie", Permoserstr. 15, 04303 Leipzig, Dipl.-Geol. R. BauDENBACHER, Naturkundemuseum Leipzig, Lortzingstr. 3 , ()4105 Leipzig, Prof. Dr. L. Eissmans,Universität Leipzig, Institut für Geophysik und Geologie, Quartärzentrum, Talstr. 35, 04103 Leipzig,

**) Für Dokumentationszwecke konnten mit freundlicher Unterstützung der Mitteldeutschen Braunkohle AG (MIBRAG) drei größere Lackprofile (Profilmeter: 16/17, 23/24 und 44/45) mit gravitativen Deformationen entnommen werden, die als Ausstellungsobjekte in die Bestände der MIBRAG, des Naturkundemuseums Leipzig und in den Bestand des Quartärzentrums am Institut für Geophysik und Geologie der Universität Leipzig eingebracht wurden.
}

Mächtige fluviatile Sande und Tone verzahnen sich mit Braunkohleflözen des Mitteleozäns bis Mitteloligozäns, gegen dessen Ende die vielgliedrige Folge der marinen bis brackischen Böhlener Schichten zum Absatz kam. Seit langen bekannt sind die reichen Blattund Fruchtfloren der Flußsandzonen und des Haselbacher Schichtkomplexes. Die besondere Attraktion des Tagebaues sind Erscheinungen der Subrosion, die einerseits zu erheblichen Mächtigkeitsanschwellungen der Braunkohle (Flöz I), andererseits zur sog. Lochbildung, d. h. tiefer Einsenkungen der Tertiärfolge ohne Mächtigkeitszunahme der Flöze geführt hat. Um eine geologische Attraktion reicher wurde (s. Abb. 2) der Tagebau, als im April/Mai 1992 bei Baggerarbeiten im 2. Abraumschnitt für kurze Zeit ein Süd-Nord-streichendes Profil im Übergangsbereich Quartär/Tertiär aufgeschlossen wurde, das auf einer Länge von ca. $75 \mathrm{~m}$ kryogen gravitative Deformationen $\left.{ }^{* *}\right)$ unterschiedlicher Morphologie und Genese aufwies (Abb. 3, 4).

Die im Tagebaubereich auftretenden gravitativen Deformationserscheinungen befinden sich im Liegenden der 1. Elstergrundmoräne und des Dehlitz-Leipziger Vorstoßbändertons (s. Abb. 1). Sie sind in Sedimenten entwickelt, die vor der ersten Elstereistransgression am Rand des Frühelstertalbodens zum Absatz kamen, in einer Position, wie sie auf Abb. 1 markiert ist, allerdings bei etwas flacherer Hanglage. Wie auch an anderen Terrassenrändern beobachtet, handelt es sich dabei um abgeschwemmte und geflossene, feinkörnige Sedimente (Schwemm- und Fließerden), die sich nach dem Tal zu mit fluviatilen Sanden und Kiesen verzahnen. Das Hangende der feinkörnigen Sedimente des Aufschlusses bildeten stellenweise geringmächtige Quarzkiese. Das Fehlen der Leitgerölle der Pleiße weist sie als umgelagerte, im konkreten Fall sogar als mehrfach umgelagerte Tertiärkiese kleiner Täler aus, die in das Frühelstertal der Pleiße mündeten. Die genannte Sedimentfolge wird diskordant von Sedimenten der glazilimnischen Randfazies (Schluff-Feinsand-Horizont bzw. "Schlepp") des Dehlitz-Leipziger Vorstoßbändertons und dem Bänderton selbst überlagert. Die brettartige, horizon- 


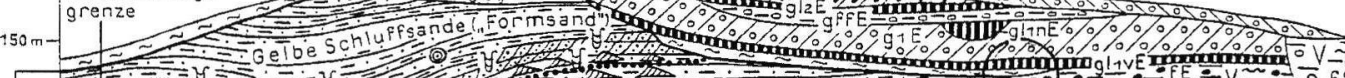

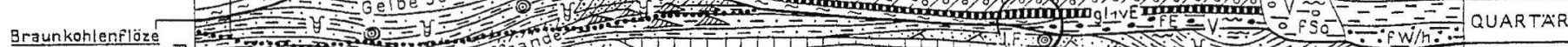

Braunkohlenflöze

Bählener ober

Flöze (I) L
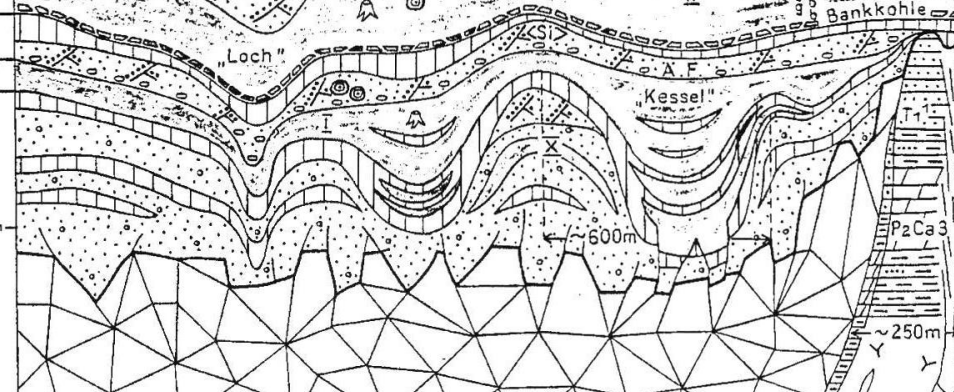

${ }_{50}^{m}$

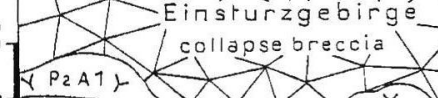

Ca.:OMNN 40

$\mathrm{P}_{2 \mathrm{~A}+2}$

(1,

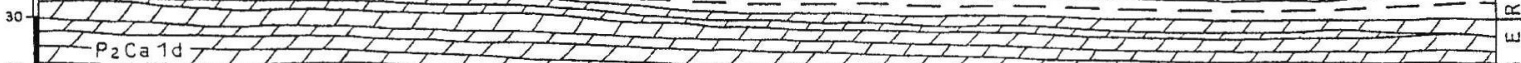

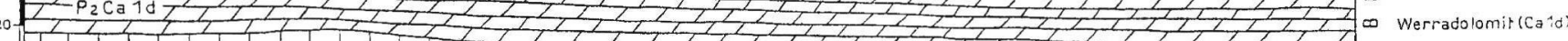

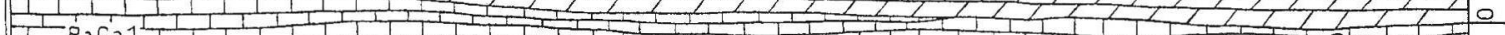

Lockersedimente

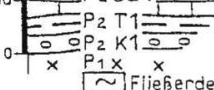

IIIIII Bänderton

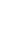

$\because$ Sand und Kie \begin{tabular}{l}
$\because-$ Sand und kies \\
\hdashline- iguartare \\
\hdashline Fluaschorter
\end{tabular}

\section{$E$ Auelehm}

- Schluff bis Feinsand,

.-- marin bis brackisch
vorwiegend Fein-und Mitre
sand, lerrestrisch

vorwiegend fein-u. Mirtelkies
stark sandig, terrestrisch
$E=-$ Schluff, Mudde, limnisch [D] Ton und roniger Schiufs,

$\square$ Braunkohle

H.Z Flora Haselbach, Zeirz

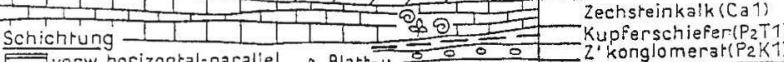

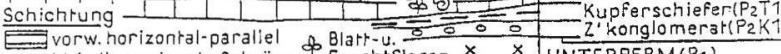
kleindimensionale Schräg. \$ Fruchtfloren $x$ x UNTERPERM(P

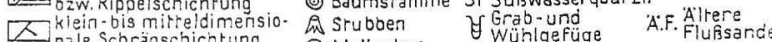

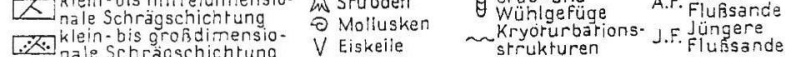




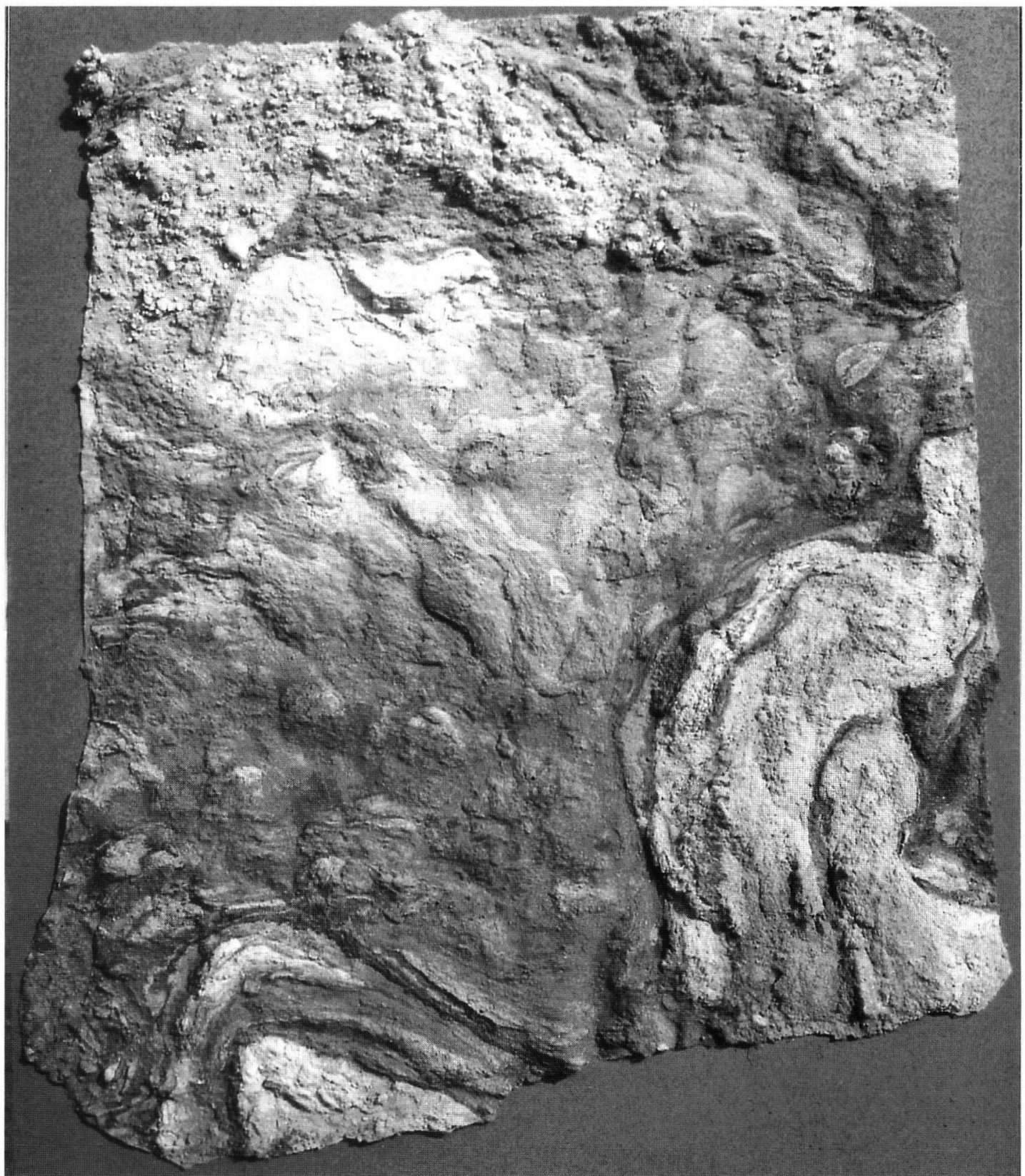

Abb. 2: Lackprofil mit gravitativen Deformationserscheinungen innerhalb feinkörniger kohliger Sedimente der "Böhlener Schichten", Tagebau Schleenhain (Profilmeter 23/24, Bildhöhe 1 m; Standort: Naturkundemuseum Leipzig).

Fig. 2: Lacquer original of gravity autoplastic sediment deformations in coaly sandy silts and silty sands from the "Böhlener Schichten", open cast lignite mine Schleenhain (profile section $\mathrm{m} 23 / \mathrm{m} 24$, altitude of figure $1 \mathrm{~m}$; exhibition place Naturkundemuseum Leipzig)

tale Lage des rhythmisch geschichteten Dehlitz-Leipziger Vorstoßbändertons auf dieser gravitativ überprägten Talfüllungssequenz läßt die Grenzfläche zwischen beiden Schichtfolgen als deutliche Erosionsdiskordanz erscheinen. Besonders eindrucksvoll war dies im Profilbereich $26 \mathrm{~m}$ bis $35 \mathrm{~m} z u$ beobachten, wo es in der unmittelbar vor dem Absatz des glazilimnischen Sediments liegenden Erosionsphase sowohl zur Abtragung der glazilimnischen Randfazies, der fluviatilen Kiese und Sande als auch zur Erosion der liegenden feinkörnigen, diapirisch aufgestiegenen Talsedimente kam. Die im Liegenden freigelegte tertiäre Schichtenfolge besteht aus stark kohlehaltigen schluffigen Feinsanden der Unteren 

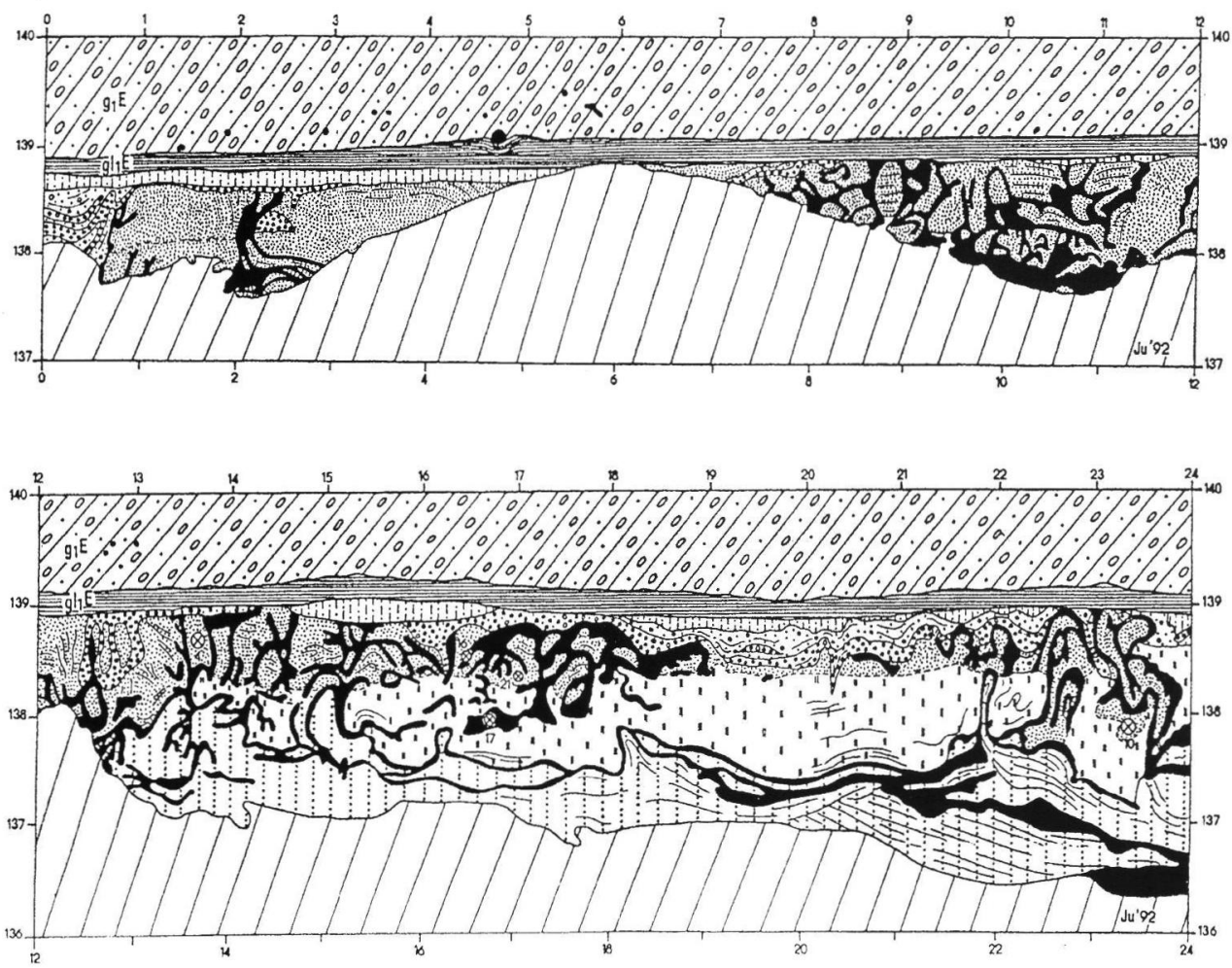

Legende:

Quartär: 1 - 1. Elstergrundmoräne,

2 - Dehlitz-Leipziger Vorstoßbänderton,

3 - Schluff-Feinsand-Horizont ("Schlepp")

4 - frühelsterglaziale, fluviatile

Sande,

5 - frühelsterglaziale Kiese,

allochthone Böhlener Schichten:

6 - Sand bis schluffiger Sand

8 - Schluffsand, stark kohlig,

Erosionsrinnenfüllung,

10 - Sandschluff bis tonig-

sandiger Schluff, stark kohlig,

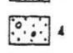

$\because 5$

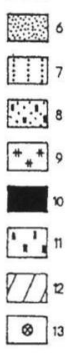

Abb. 3: Kryogene und gravitativ autoplastische Schichtdeformationen im Liegenden des Dehlitz-Leipziger Vorstoßbändertons, gezeichnet nach Naturbefund. Braunkohlentagebau Schleenhain südlich Leipzig, April/Mai 1992.

Fig. 3: Cryogenic and gravity autoplastic sediment deformations in subjacent beds of Dehlitz-Leipzig varved clay, drawn in the field

Open cast lignite mine Schleenhain southern of Leipzig, April/May 1992.

(Der in der Zeichnung durch die Signatur entstehende Eindruck des Schichtauskeilens der Basislagen des Dehlitz-Leipziger Vorstoßbändertons entspricht nicht dem Naturbefund. Die Basislagen des Bändertons greifen diskordant über den gesamten Untergrund hinweg.)

Böhlener Schichten. Die Sedimente dieses Teils, wie auch die im Quartär umgelagerten tertiären feinkörnigen Talsedimente sind im Profil lithologisch deutlich differenziert und granulometrisch gliederbar (Abb. 5).

Die Unteren Böhlener Schichten werden im untersuchten Profil (Abb. 5) durch eine enge Wechsellagerung im cm- bis dm-Bereich von grau bis bräun- lichgrauen Schluffsanden und hell- bis dunkelbraunen tonigen Sandschluffen vertreten. Die Schluffsande (Legende-Nr. 7 in Abb. 3) weisen in den untersuchten Proben bei einer mittleren Korngröße von $75 \mu \mathrm{m}$ bindige Anteile $<63 \mu \mathrm{m}$ von $45 \%$ bis $49 \%$ auf. Ihr Sortierungsgrad nach TRASK erweist sich als schlecht (3,1 bis 3,5). Im südlichen Profilteil ( $\mathrm{m} 12$ bis m34) entwickeln sich diese Schluffsande zu einem 


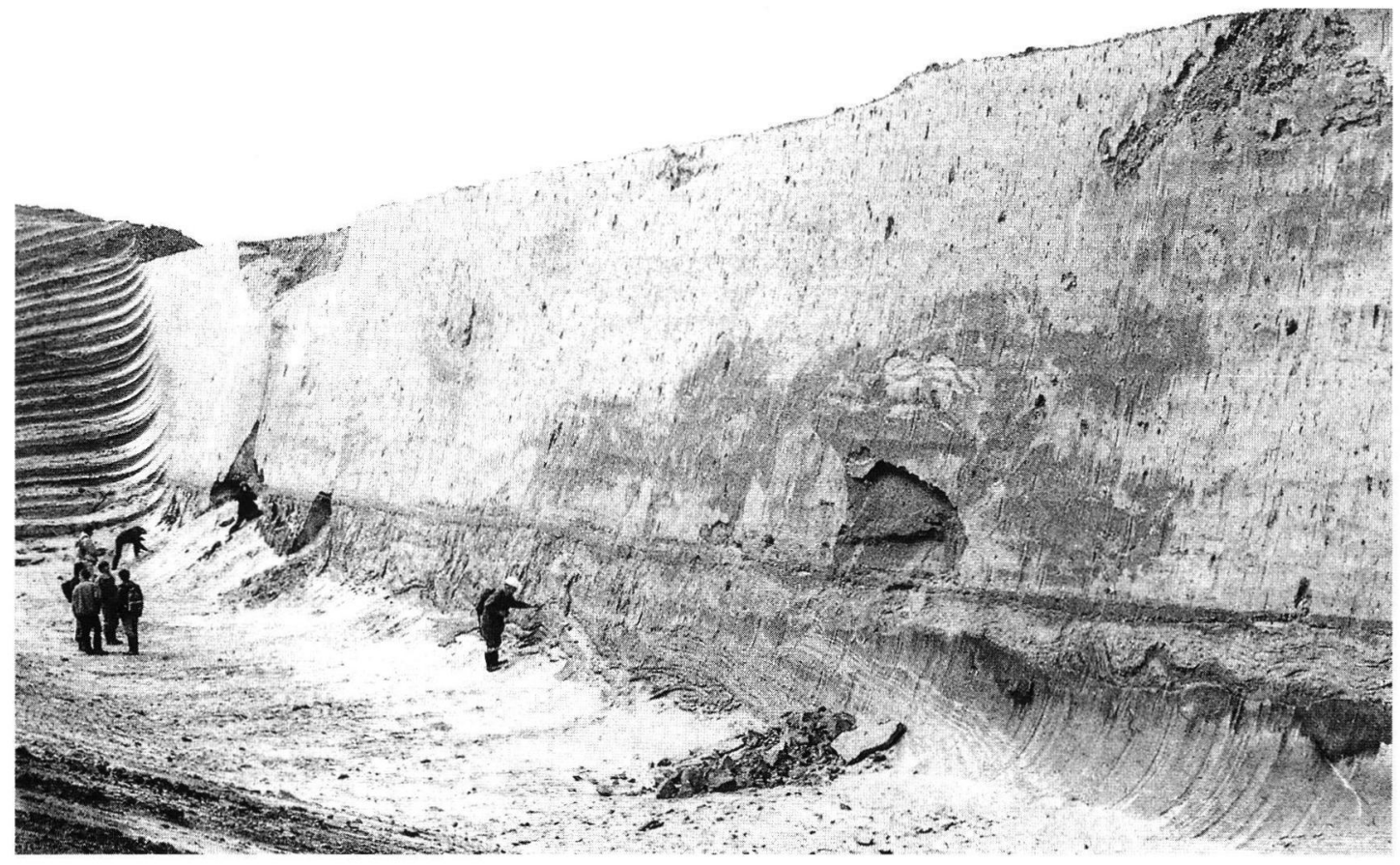

Abb. 4: Überblick über die unter 1. Elstergrundmoräne und Dehlitz-Leipziger Bänderton im Tagebau Schleenhain auftretenden gravitativ deformierten Talfüllungssedimente (Bildbreite ca. $50 \mathrm{~m}$ ). Man beachte die innerhalb der ca. $8 \mathrm{~m}$ mächtigen Grundmoräne auftretenden glazigenen Schollen und Wirbelstrukturen ("Walzen").

Fig. 4: Overview of the gravity deformed valley sediments situated in the subjacent bed of first Elsterian till and Dehlitz-Leipzig varved clay, open cast lignite mine Schleenhain / figure width approximately $50 \mathrm{~m}$ ).

Note the glacigenic blocks and vorticity structures situated in the first Elsterian till ( 8 metres in thickness ).

mächtigen Paket mit deutlicher Schichtung im cm-Bereich. Die mit ihnen in Wechsellagerung stehenden tonigen und z. T. stark kohligen Sandschluffe (Legende-Nr. 9 in Abb. 3) zeigen bei einer ebenfalls schlechten bis sehr schlechten Sortierung (3,9 bis 4,7) eine mittlere Korngröße von $23 \mu \mathrm{m}$ und bindige Anteile von $64 \%$ bzw. $68 \%$. Weiterer Bestandteil dieser Wechsellagerung sind stark kohleführende, dunkelbraune Sandschluffe bis tonig-sandige Schluffe (Legende-Nr. 10 in Abb. 3), die als dünne Lagen sowohl im unteren Teil als auch innerhalb der quartären Talfüllungssequenz auftreten und intensiv deformiert erscheinen. Medianwerte von $16 \mu \mathrm{m}$ bis $34 \mu \mathrm{m}$, bindige Anteile von $50 \%$ bis $87 \%$ sowie eine mittelmäßige bis schlechte Sortierung $(2,9$ bis 3,4$)$ kennzeichnen dieses Sediment. Im Hangenden dieser Wechsellagerung folgt ein bis ca. 1,5 m mächtiges, kompaktes, ungeschichtetes Paket tonig-sandiger bis toniger Schluffe (Legende-Nr. 11 in Abb. 3) von dunkelbrauner bis schwarzer Farbe, das durch eine deutliche Kohleführung auffällt. Diese zeigen bei Medianwerten von $6 \mu \mathrm{m}$ bis $18 \mu \mathrm{m}$ bindige Anteile von $>77$ $\%$ und ebenfalls eine mittelmäßige bis schlechte Sortierung (2,3 bis 3,9) und bilden die Basis der quartären Talfüllungssequenz. Darüber folgen im Profil weiße bis rötlich-graue Mittel- bis Grobsande mit bindigen Anteilen $<63 \mu \mathrm{m}$ zwischen $9 \%$ und $23 \%$ (Legende-
Nr. 6 in Abb. 3). Sie zeigen eine offensichtlich auf Umlagerungsprozessen begründete deutlich bessere Sortierung (1,6 bis 2,4). Im Profilbereich $\mathrm{m} 66$ bis $\mathrm{m} 72$ ist eine Erosionsrinne aufgeschlossen, die von dunkelbraunen, kohligen Schluffsanden mit deutlichem Anlagerungsgefüge eingenommen wird (Legende- $\mathrm{Nr}$. 8 in Abb. 3). Diese Schluffsande besitzen bindige Anteile von $18 \%$ bis $32 \%$ und eine ebenfalls sehr schlechte Sortierung ( 3,4 bis 5,3$)$.

\section{Beschreibung der gravitativen Deformationsstrukturen}

\subsection{Absinkungsstrukturen infolge gravitativ bedingter Dichtesaigerung}

Modellbeispiel für die an eine schwerkraftbedingte Trennung unterschiedlicher Sedimente nach der Dichte gebundene Deformation stellen die in den quartären Schichten des Leipziger Raumes mehrfach beschriebenen Taschen- und Tropfenböden dar (EIssMANN 1985, 1987). Der dabei ablaufende Saigerungsprozeß, der zu einem gravitativen Absinken eines dichteren Substrats in ein weniger dichtes Substrat führt, hält solange an, bis die dabei auftretende Reibung größer bzw. gleich der Dichtedifferenz zwi- 

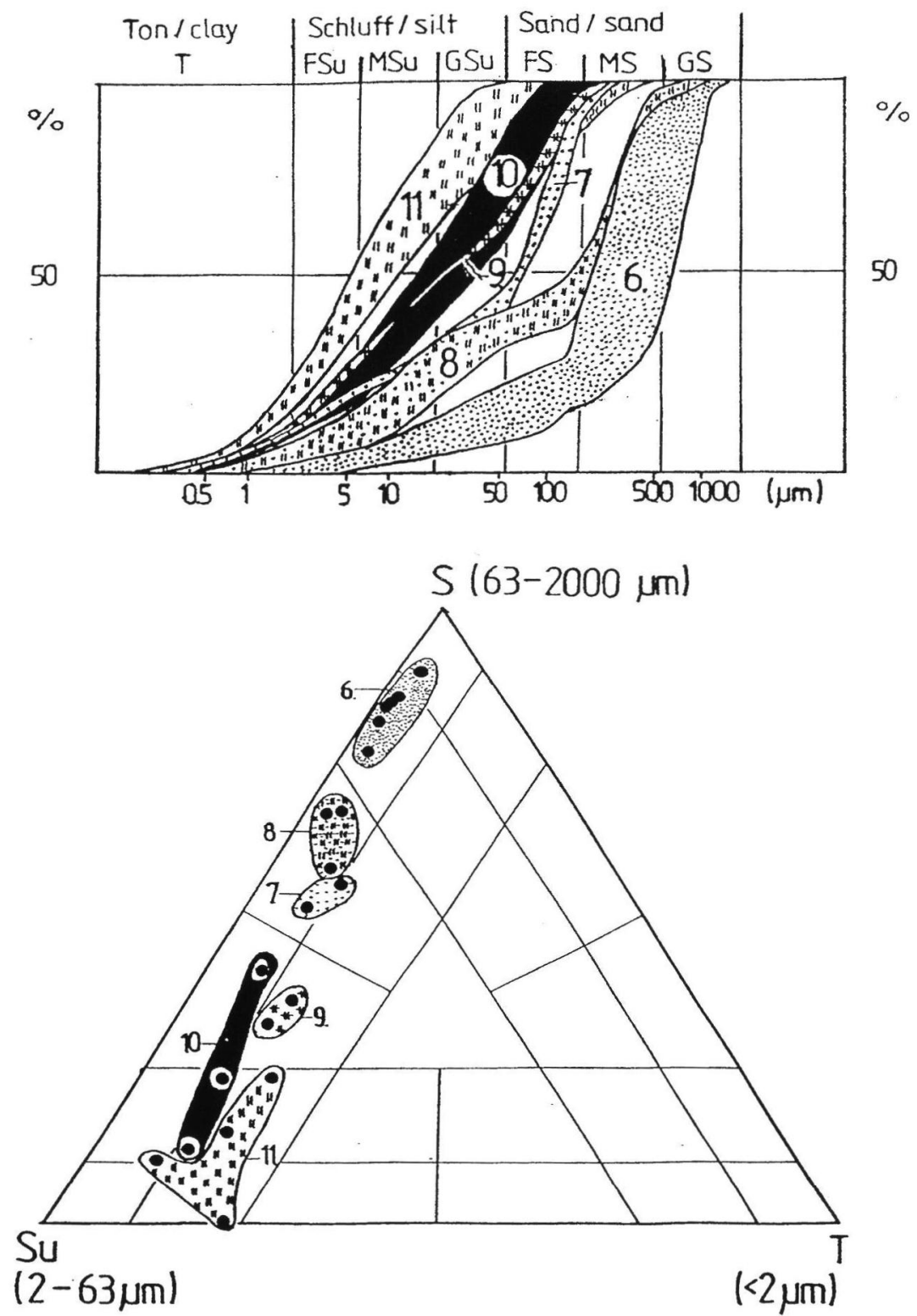

Abb. 5: Summenkurvenstreubreiten und Korngrößenklassifikationsdiagramm nach MüLLER (1961) der untersuchten Sedimente im Profil.

(Die Nummern entsprechen den Legende-Nr. in der Abb. 3.)

Fig. 5: Range of cumulative curves and grain size classification diagram (after MüLLER, 1961) of the investigated sediments in the profile.

(The numbers correspond to the legend numbers in figure 3)

schen den betrachteten Sedimenten ist. Während des Saigerungsprozesses ist die mechanische Festigkeit des Sediments so stark herabgesetzt, daß ein kontinuierlicher Materialaustausch mit einer damit verbundenen Wanderung des verdrängten leichteren Substrats in die freiwerdenden Räume stattfinden kann (Eissmann, 1981). Derartige Bedingungen sind in den sommerlichen, wassergesättigten
Auftauböden periglaziärer Gebiete gegeben. Im untersuchten Profil waren Strukturen eines derartigen Prozesses besonders im Profilbereich m8-m19 (Abb. 3) zu beobachten. Dabei ist innerhalb der Talfüllungssequenz ein "Zertropfen" kohliger Sandschluffe in Grobsande, z. T. auch in tonige Schluffe zu beobachten. Tropfenartige Strukturen ( $\mathrm{m} 17 / \mathrm{m} 18$; Abb. 6; $\mathrm{m} 28 / \mathrm{m} 29$, Abb. 8) mit einem gewundenen, netzar- 


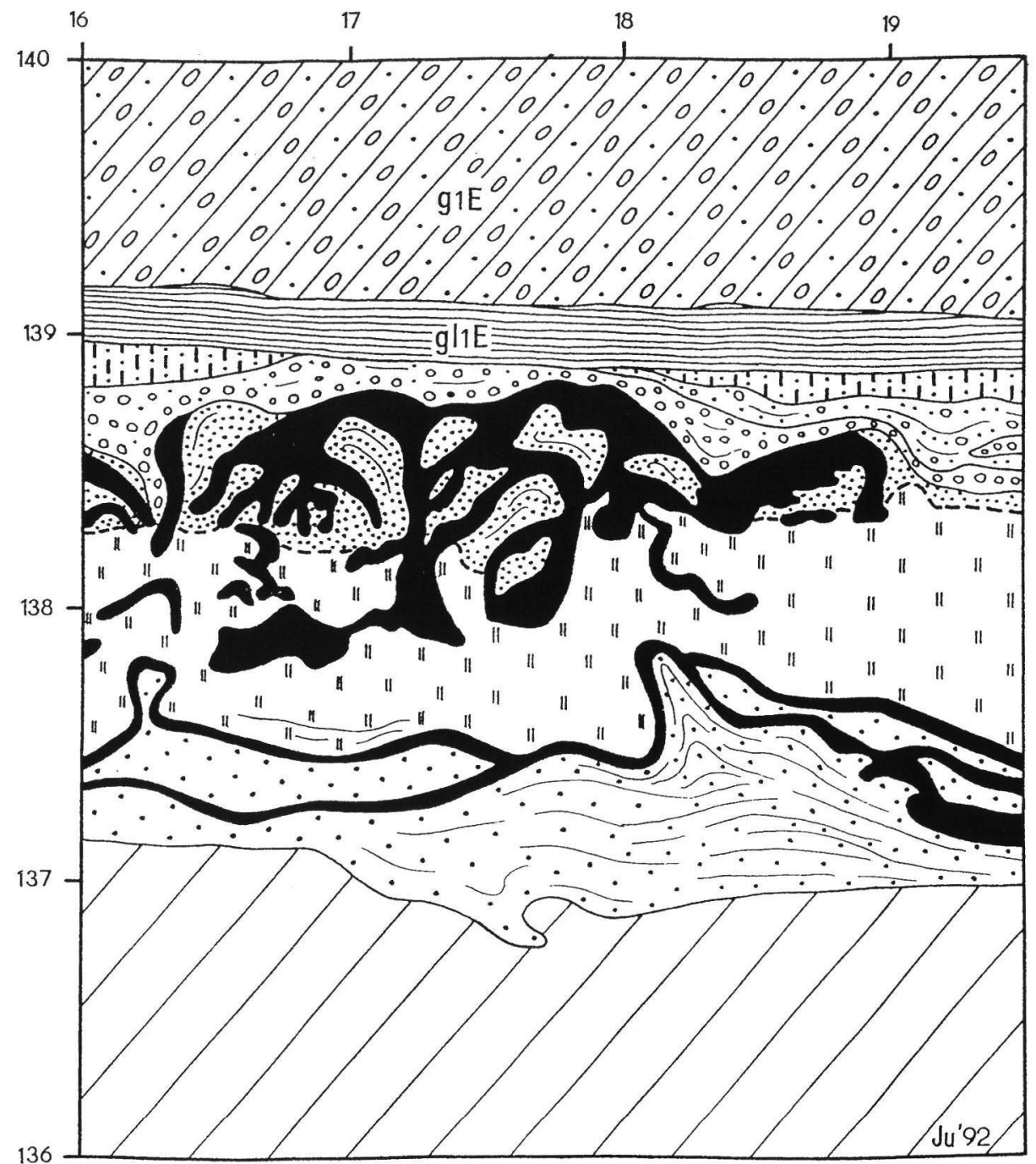

Abb. 6: Tropfenartige Absinkungsstruktur kohliger Sandschluffe in Grobsande bzw. tonige Schluffe. (Profilausschnitt m16 - m19,5; Legende siehe Abb. 3)

Fig. 6: Drop-shaped gravity sinking structure of coaly sandy silts in coarse sands and clayey silts.

(Profile section $\mathrm{m} 16$ - $\mathrm{m} 19,5$, legend see figure 3)

tigen Verlauf ihrer Hälse (m8-m12), bis hin zur völligen Auflösung der Schichten sind die Folge. Unmittelbar vergleichbar sind diese Strukturen mit dem von EIsSMann (1975) beschriebenen Taschen- und Tropfenboden an der Basis des Hohendorfer Beckens, wo "kohlehaltiger, brauner Schluff 0,4 $\mathrm{m}$ bis 0,6 $\mathrm{m}$ tief in unterlagernde Sedimente aus feinsandigen Mittel- und Grobsand eingesunken ist". Interessant ist, daß bei den im Profil beobachteten gravitativ bedingten $\mathrm{Ab}$ sinkungsstrukturen einerseits ein Einsinken von feinkörnigerem Material in grobkörnigeres (Sandschluf- fe in Grobsande) und andererseits auch der umgekehrte Fall (Sandschluffe in tonige Schluffe) zu beobachten ist.

\subsection{Auflastbedingte Absinkungsstrukturen}

Unter den Bedingungen einer erhöhten Schichtauflast auf einem wassergesättigten, mobilen Untergrund kann es zu einem Absinken der auflagernden Schicht und damit zur Verdrängung und zu Ausgleichsbewegungen der unterlagernden Schichten kommen. Der 
S

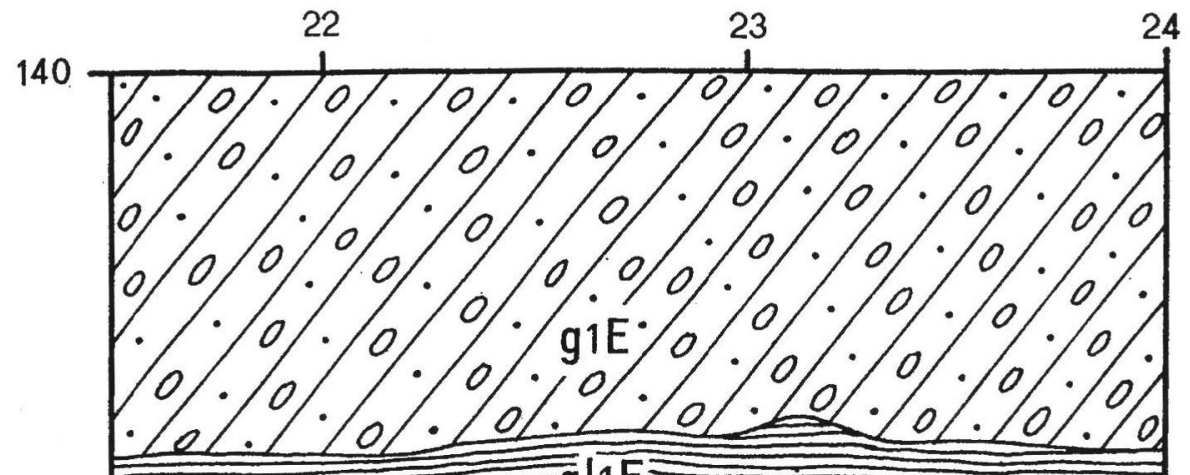

139
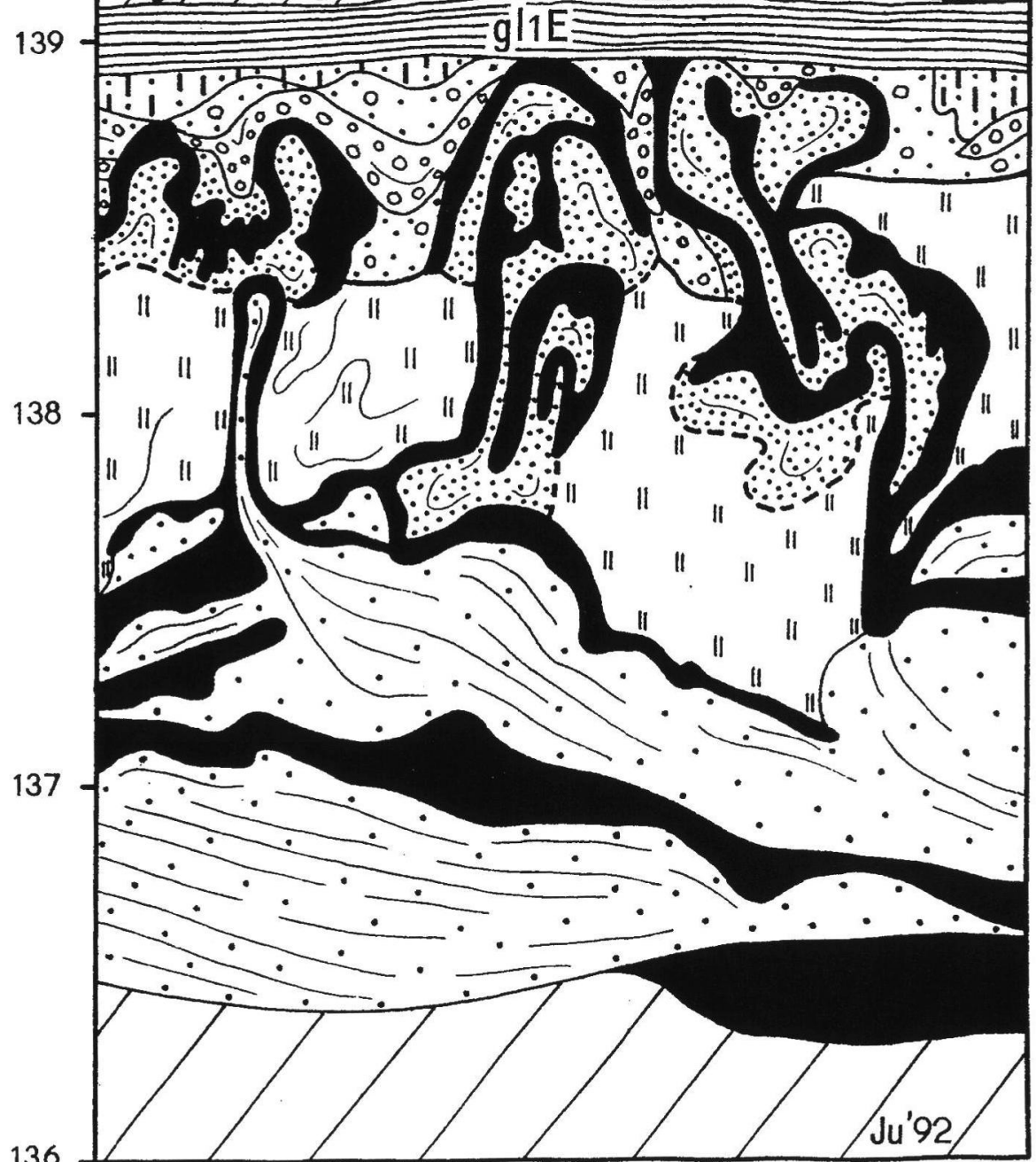

Abb. 7: Schichtaufwulstungen und diapirartige Aufstiegsstruktur wechsellagernder Schluffsande und kohliger Sandschluffe der unteren Böhlener Schichten in hangende Profilbereiche. (Profilausschnitt m21,5 - m24; Legende siehe Abb. 3)

Uparching of sediments and a diapiric ascending structure of interbedded silty sands and coaly sandy silts in the upper part of the "Untere Böhlener Schichten". 


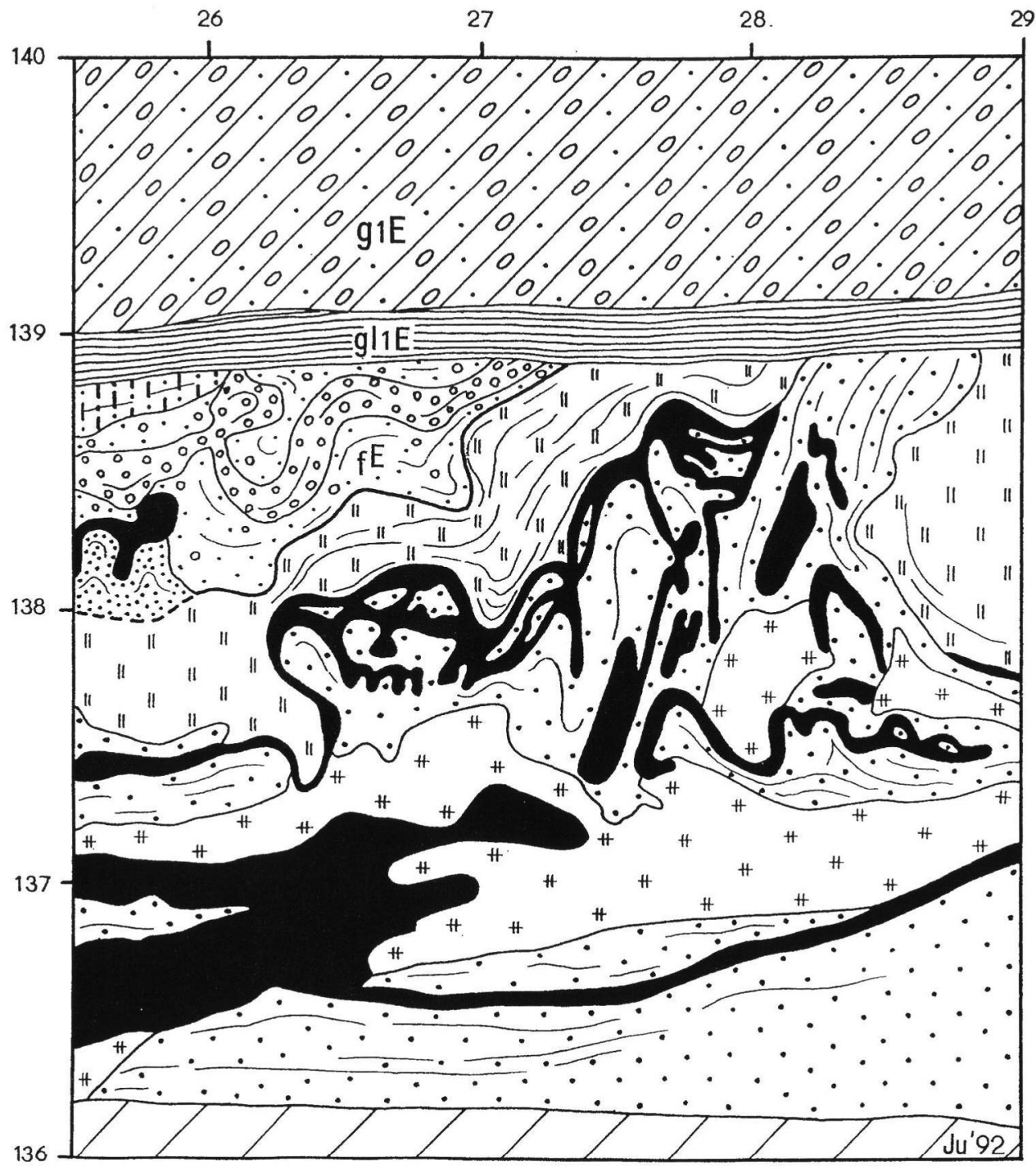

Abb. 8: Tropfenartige Absinkungsstruktur kohliger Sandschluffe innerhalb einer gravitativ aufgestiegenen Folge von Schluffsanden und tonigen Schluffen.

(Mit dem Aufstieg der Sedimente ist ein Hochschleppen der hangenden fluviatilen Kiese verbunden. Die gesamte Folge wird von einer deutlichen Erosionsdiskordanz im Hangenden begrenzt. Profilausschnitt m25,5 - m29; Legende siehe Abb. 3)

Fig. 8: Drop-shaped gravity sinking structure of coaly sandy silts situated in a gravitational ascending sequence of silty sands and clayey silts.

(The gravity ascending of sediments causes an upward drag of the hanging fluviatile gravels. The total sequence is limited by a clear erosion unconformity in the stratigraphical hanging. Profile section $\mathrm{m} 25,5-\mathrm{m} 29$, legend see figure 3.)

Prozeß der auflastbedingten Schichtverdrängung führt zur Bildung von taschen- und sackartigen Strukturen der auflagernden Schicht und initiiert ein Abströmen des verdrängten Substrats im Untergrund in Bereiche geringerer Belastung. Oft ist anhand der in den Profilen gefundenen Formen eine eindeutige Trennung zwischen auflastbedingten Verdrängungsprozessen und Verdrängungsprozessen infolge gravitativer Dichtesaigerung nicht möglich. Vielfach stehen beide Pro- zesse in enger Wechselwirkung, die oft durch das gemeinsame Auftreten klassischer Tropfenböden mit den von EISSMANN $(1981,1987)$ beschriebenen Girlandenböden und Klein-Polygonen demonstriert wird.

Als auflastbedingte Deformation kann im untersuchten Profil die Bildung von Taschen des "Schlepps" und der fluviatilen Sande und Kiese in die unterlagernden feinkörnigen Talsedimente angesehen werden 


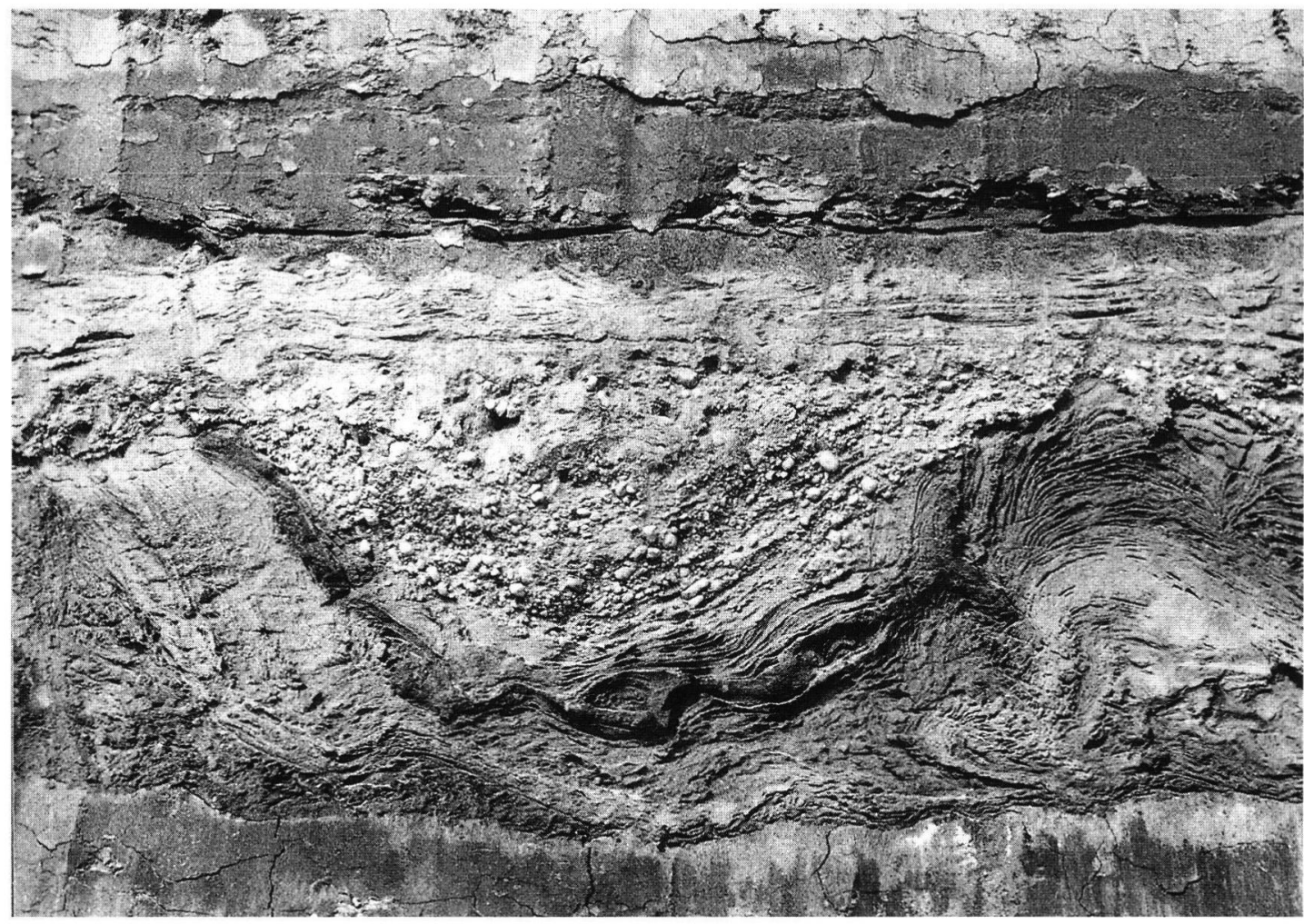

Abb. 9: "Tasche" fluviatiler Sande und Kiese innerhalb feinkörniger Sedimente der "Böhlener Schichten", Tagebau Schleenhain (Detailausschnitt Profilmeter 43-45, Bildhöhe $1,5 \mathrm{~m}$ ).

Fig. 9: "Pocket structure" of fluviatile sands and gravels in fine-grained sediments of "Böhlener Schichten", open cast lignite mine Schleenhain (detail section of profile $\mathrm{m} 43-\mathrm{m} 45$, altitude of figure $1,5 \mathrm{~m}$ ).

(s. Abb. 9). Besonders eindrucksvoll ist dies in den Profilbereichen m12-m13, m33-m34 und m43-m45 zu sehen (Abb. 3, Abb. 8). Einsinkungsbeträge der Schichten bis maximal $1 \mathrm{~m}$ sind zu beobachten. Interessant ist, daß diese Taschen zum einen nur mit fluviatilen Sanden und Kiesen (z. B. m12/m13, m43/m45) gefüllt sind und zum anderen, daß sie in schalenförmiger Anordnung zusätzlich noch mit Sediment des SchluffFeinsand-Horizontes ("Schlepp") ausgekleidet sind (z. B. m33/m34).

\subsection{Aufstiegsstrukturen der freien Solikinese}

Gravitativ-autoplastische Fließprozesse können einerseits initiiert werden als Folge der Existenz größerer Belastungsunterschiede (schwankendes Mächtigkeitsverhältnis) zwischen hangenden und liegenden Schichten eines mobilen Sedimentpaketes und andererseits entstehen sie als Folge des Sedimentumbaus beim Prozeß der Dichtesaigerung (Freie Solikinese). Beide Antriebsmechanismen führen zu Ausgleichsbewegungen mobiler Schichten im Untergrund. Dabei entstehen Schichtaufwulstungen, diapirartige Aufstiegsstrukturen, und mitunter kommt es auch, in Abhängigkeit von den vorhandenen Dichteunter- schieden, der Reibung und der Mächtigkeit der überlagernden Schicht, zum Durchbrechen von Sedimentfolgen (Diapirismus).

Innerhalb des Profils betrifft dies den Aufstieg der wechsellagernden tertiären Sedimente der Unteren Böhlener Schichten in die hangenden Bereiche (s. Abb. 10). Als aktives Medium sind dabei Schluffsande (Legende-Nr. 7, Abb. 3) anzusehen, die die mit ihnen in Wechsellagerung stehenden hangenden, feinkörnigeren Sedimente (Sandschluffe, tonig-kohlige Schluffe) mitschleppen. Dabei kommt es zur Entstehung dom- bis pilzartiger Strukturen (m22, m30, Abb. 3 und 7) und in Ausnahmefällen zu einem mehr oder weniger intensiven Durchbrechen der hangenden Folge (m27-m29, Abb. 8).

\section{Synthese}

Das Auftreten der beobachteten gravitativen Deformationsstrukturen im Liegenden der Sedimente des ältesten Eisvorstoßes im Saale-Elbe-Gebiet macht ihre nichtglazigene Bildung deutlich. Die Deformation erfolgte weitgehend gravitativ in Auftauböden der frü- 


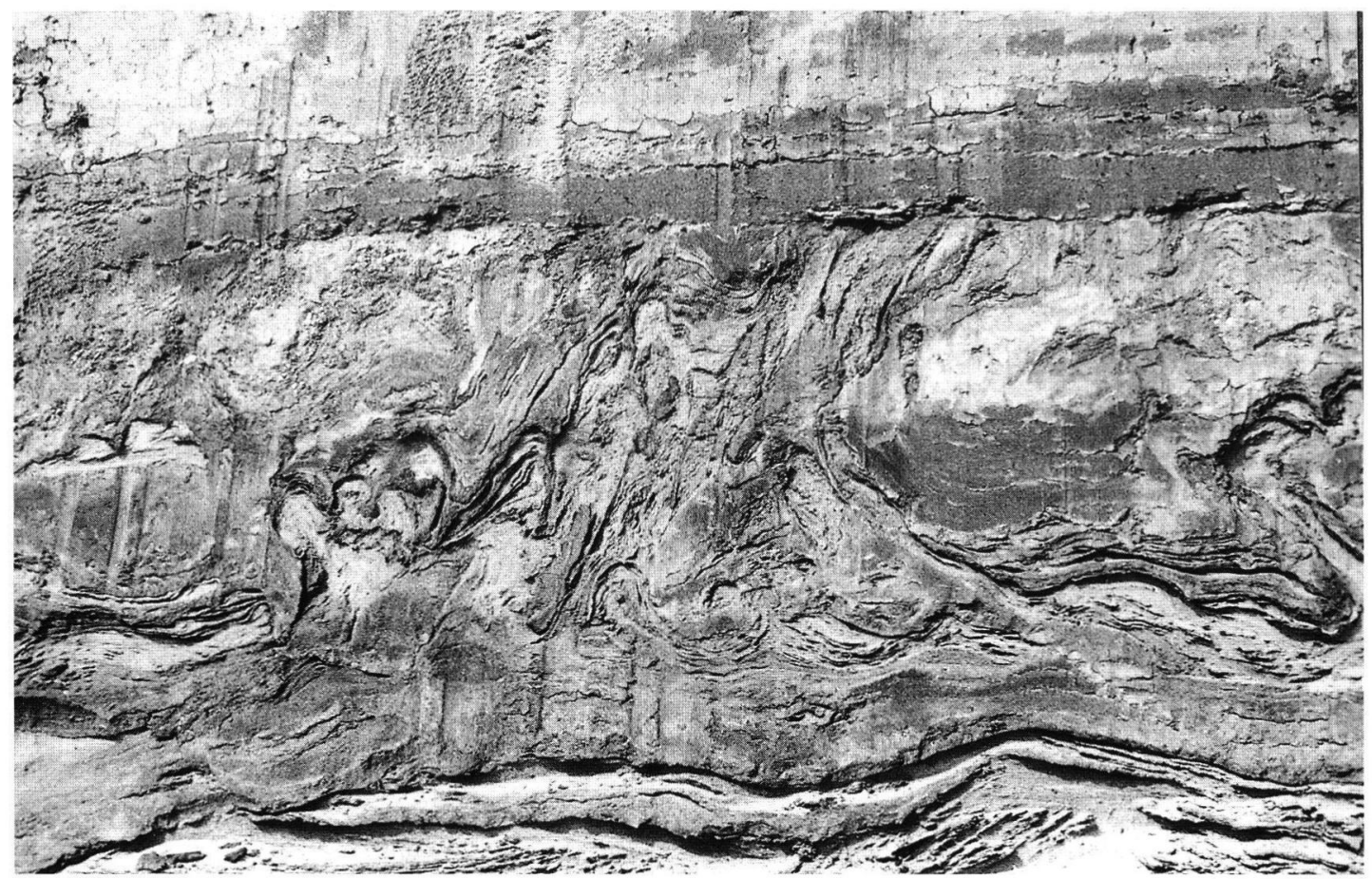

Abb. 10: Gravitative Aufstiegsstrukturen feinkörniger Sedimente der "Böhlener Schichten" in hangende Bereiche, Tagebau Schleenhain (Detailausschnitt Profilmeter 25-30, Bildhöhe 3m)

Fig. 10: Diapiric ascending structures of silty sandy sediments in the upper part of the "Böhlener Schichten", open cast lignite mine Schleenhain (detail section of profile $\mathrm{m} 25-\mathrm{m} 30$, altitude of figure $3 \mathrm{~m}$ ).

hen Elstereiszeit bis zu Beginn des Eisvorstoßes in der Leipziger Tieflandsbucht. Vor der Sedimentation des rhythmisch geschichteten Dehlitz-Leipziger Bändertons war sie abgeschlossen. Der aus der Lagerung der Schichten im untersuchten Profil abzuleitende zeitliche Ablauf läßt sich folgendermaßen beschreiben: Im jüngeren Tertiär und frühen Quartär verfielen die älteren Tertiärsedimente bis in das Niveau der Unteren Böhlener Schichten der Abtragung. In den Anaglazialphasen der frühen Kaltzeiten kamen die feinkörnigen Tertiärsedimente an den Talrändern streifenweise wieder zum Absatz. Wie das beschriebene Beispiel aus der frühen Elstereiszeit demonstriert, wechselten Phasen der Akkumulation und Erosion. Den Abschluß der deluvial-fluviatilen Talrandfazies bilden quarzreiche kiesig-sandige Sedimente. Die Zeit der danach einsetzenden glazilimnischen Sedimentation ("Schlepp" bzw. Schluff-Feinsand-Horizont) im Vorfeld des nahenden Inlandeises war durch einen Wechsel von Dauergefrornis und Frostbodendegradation gekennzeichnet. In Stadien des Frostbodenzerfalls kam es bei Wassersättigung innerhalb der oberen Auftauschicht, die von den Sedimenten der Talfüllungssequenz eingenommen wurde, zu labilen Dichteunterschieden. Diese führten zu den beschriebenen, kryogen verursachten gravitativen Sedimentausgleichsbewegungen. Dabei spiegeln die trop- fen- bis netzartigen Absinkungsstrukturen der kohligen Sandschluffe $(\mathrm{m} 17 / \mathrm{m} 18, \mathrm{~m} 28 / \mathrm{m} 29$, Abb. 6 und 8) offensichtlich eine ältere Phase dieser kryogenen Deformation wider. Als vergleichsweise jüngere kryogene Deformation ist die im Profil beobachtete Taschenbildung der fluviatilen Kiese und Sande (m12/m13, m43/m45) anzusehen. Die unterschiedliche Füllung dieser Taschen läßt auf ihre Bildung noch während der Sedimentation der glazilimnischen Randfazies schließen. Als zeitlich jüngste kryogene Deformation im untersuchten Profil ist der beobachtete Aufstieg der feinkörnigen Sedimente der Talfüllungssequenz anzusehen. Diese wahrscheinlich durch Unterschiede in der Sedimentauflast bedingten Ausgleichsbewegungen waren z. T. von einem Aufschleppen der hangenden kiesigen Bereiche begleitet $(\mathrm{m} 27 / \mathrm{m} 29)$. Abgeschlossen wurden sämtliche beobachteten, ins Anaglazial der Elstervereisung zu stellenden kryogenen Deformationserscheinungen von einer deutlich sichtbaren (? lokalen) Erosionsphase. Diese örtlich noch in der Sedimentationszeit der glazilimnischen Randfazies einsetzende Abtragung erfaßte die hangenden Bereiche der kryoturbat gestörten Folge. Der rhythmisch geschichtete Dehlitz-Leipziger Vorstoßbänderton liegt diskordant auf dieser im Anaglazial durch Frostwechselprozesse deformierten periglaziären Sedimentunterlage 


\section{Danksagung}

Bei der Mitteldeutschen Braunkohle AG (MIBRAG) möchten wir uns für die Möglichkeit der Bearbeitung und insbesonders bei Herrn Geol.-Ing. Th. Fischkandl für die Unterstützung bei der Probenahme und Lackprofilherstellung bedanken. Für die zeichnerische Fertigstellung der Profile danken wir Herrn H. Bulnheim recht herzlich.

\section{Schriftenverzeichnis}

BelLmaN, H.-J. und R. STARKE (1978): Ergebnisse geologischer und tonmineralogischer Untersuchungen an den Formsanden der Leipziger Bucht.- In: Wiss. Ztschr. ErnstMoritz-Arndt-Univ. Greifswald, Math.-Naturwiss. R., XXVII, Beitr. Miner. Technol. Tone: 7-19, Greifswald BöHмE, P. (1962): Lagerstätten der Steine und Erden im Deckgebirge des nordwestsächsischen Braunkohlenreviers. Braunkohletagebau Schleenhain.- In: Exkursionsführer Geol. Ges. DDR: Das Pleistozän im sächsisch-thüringischen Raum: 73-80
EISSMANN, L. (1987): Lagerungsstörungen im Lockergebirge. Exogene und endogene Tektonik im Lockergebirge des nördlichen Mitteleuropa.- Geophys. Veröff. Univ. Leipzig, III, 4: 7-77

EIssmann, L., O. Priese und E. Richter (1985): Die Geologie des Naherholungsgebietes Kulkwitz-Miltitz bei Makranstädt. Ein Leitprofil des Glaziärs und Periglaziärs in Sachsen.- Abh. Ber. Naturkundemuseum Mauritianum, 11, 3: 217-248, Altenburg

EIssmann, L. (1984): Beiträge zur Periglazialgeologie des Saale-Elbe-Gebietes. I. Der Tropfboden von Süptitz, Kreis Torgau".- Abh. Bcr. Naturkundemuseum Mauritianum, 11, 2: 106-113, Altenburg

Eissmann, L. (1981): Periglaziäre Prozesse und Permafroststrukturen aus sechs Kaltzeiten des Quartärs. Ein Beitrag zur Periglazialgeologie aus der Sicht des Saale-Elbe-Gebietes.- Altenburger Naturwiss. Forsch., 1: 1-171, Altenburg

EIssmanN, L. (1975): Das Quartär der Leipziger Tieflandsbucht und angrenzender Gebiete um Saale und Elbe.Schriftenreihe Geol. Wiss, 2 ,

Müller, G. (1961): Das Sand-Silt-Ton-Verhältnis in rezenten marinen Sedimenten.- N. Jb. Min.: 148-163

Manuskript eingegangen am 6.10 .1993 